\title{
Higher-Order Methods for Compressible Turbulent Flows Using Entropy Variables
}

\author{
Laslo T. Diosady*and Scott M. Murman ${ }^{\dagger}$ \\ NASA Ames Research Center, Moffett Field, CA, USA
}

\section{Introduction}

Turbulent flows have a large range of spatial and temporal scales which need to be resolved in order to obtain accurate predictions. Higher-order methods can provide greater efficiency for simulations requiring high spatial and temporal resolution, allowing for solutions with fewer degrees of freedom and lower computational cost than traditional second-order computational fluid dynamics (CFD) methods. ${ }^{1}$ Higher-order methods have been widely used for turbulent flows. However, the reduced numerical stabilization present in higher-order schemes implies that special care needs to be taken in the development of numerical methods to suppress nonlinear instabilities. ${ }^{2-6}$ In this work we present the development of a higher-order space-time discontinuous Galerkin method with a focus on the aspects of our numerical scheme required for ensuring nonlinear stability for turbulent simulations at high Reynolds numbers.

The importance of nonlinear stability was recognized in the first direct numerical simulation (DNS) of incompressible isotropic turbulence using spectral methods. ${ }^{2}$ The quadratic nonlinearities in the inviscid fluxes lead to aliasing errors when using a collocation or pseudo-spectral scheme. Over long-time evolution these aliasing errors can lead to non-physical accumulation of energy and ultimately catastrophic failure of the numerical simulation. Evaluating the fluxes in the physical space using a dealiasing rule with $3 / 2 \mathrm{~N}$ points results in a Galerkin formulation (i.e. exact projection of the fluxes) and eliminates the aliasing errors. In particular, the kinetic energy is discretely bounded provided a symplectic time integration scheme is used, resulting in a discretely nonlinearly stable scheme. ${ }^{7}$ An alternative approach to ensure discrete nonlinear stability for incompressible Navier-Stokes simulations is to formulate the inviscid fluxes in "skew-symmetric" form. ${ }^{3}$ This form also ensures the discrete conservation of kinetic energy in the absence of viscosity, while ensuring discrete conservation of mass and momentum. This skew-symmetric formulation has been widely used for simulations of turbulent flows using spectral methods as well as non-dissipative central-difference schemes.

For the compressible Navier-Stokes equations the development of nonlinearly stable schemes is complicated by the fact that the nonlinear fluxes are rational functions of the conservative variables. The situation is further complicated by the presence of shocks, though for the present discussion we consider only subsonic flows. Multiple avenues have been pursued for the development of nonlinearly stable schemes for compressible flows, which we discuss in the following paragraphs.

Upwind schemes, based on approximate Reimann solvers are widely used for steady compressible flow simulations. While these scheme have been used for DNS of compressible flows, the 2nd-order formulations typically used have too much numerical dissipation, and can result in poor predictions in the case of marginally resolved DNS or large eddy simulations (LES).

Non-dissipative central difference schemes have also been widely used for the simulations of turbulent compressible flows. Non-linear instabilities may be controlled using higher-order filtering schemes or hyperviscosity methods, however these may introduce non-physical dissipation. ${ }^{8}$ Along the lines of approaches used for incompressible flows, skew-symmetric formulations have been developed for non-dissipative schemes which attempt to globally conserve either entropy or kinetic energy in the absence of viscosity or shocks. ${ }^{4-6,8,9}$ However, such skew-symmetric schemes do not always ensure discrete conservation of both energy and

\footnotetext{
*Postdoctoral Fellow, Oak Ridge Associated Universities, laslo.diosady@nasa.gov

†Scott.M.Murman@nasa.gov
} 
entropy. Additionally, the effectiveness of such schemes may be limited to moderate Reynolds numbers. ${ }^{8}$ Furthermore, to the authors knowledge, extensions to finite element formulations do not exist.

An alternative approach to develop nonlinearly stable central-difference schemes has been to use locally adapted stencils (i.e. limiters or WENO) schemes. A particularly promising approach uses a comparison technique to develop a WENO scheme for a summation-by-parts simultaneous approximation term (SBPSAT) finite-difference method, which is discretely entropy stable. ${ }^{10}$ Unfortunately, extension of this approach to general unstructured, finite element formulations is unclear.

For higher-order finite-element discretizations of compressible flows, nonlinear instabilities have been suppressed by using more accurate quadrature rules for the integration of the nonlinear fluxes. ${ }^{11}$ Such "polynomial-dealiasing" attempts to directly address the inexact projection of the nonlinear fluxes using collocated quadrature rules and has been successfully applied with higher-order discontinuous Galerkin discretizations at moderate Reynolds numbers. ${ }^{11,12}$ However, as we have previously demonstrated, this approach is not general. ${ }^{13}$

Provably entropy-stable formulations for compressible flows have been developed based on stabilized continuous and discontinuous Galerkin finite-element methods discretized using entropy variables. ${ }^{14,15}$ Despite their rigorous mathematical underpinning, finite-element formulations using entropy variables have been less widely used, in part, because they cannot be combined with explicit time-marching schemes and are viewed as too expensive. Additionally, low-order simulations have suggested the benefits of the entropy variable formulations are largely theoretical, with little practical difference to conservative variables. ${ }^{16}$ In this work we demonstrate that for higher-order simulations of turbulent compressible flows, a non-linearly stable entropy variable formulation is necessary in order to maintain stability for marginally or under-resolved simulations.

We present a higher-order space-time discontinuous-Galerkin (DG) finite-element method for the simulation of turbulent compressible flow. Through numerical experiments we assess the nonlinear stability of different formulations using collocated and dealiased schemes with conservative or entropy variables. We demonstrate that with increasing Reynolds number and polynomial solution order, nonlinear stability is achieved only through a formulation consistent with a provably-stable formulation. Furthermore, we demonstrate that such a scheme may be implemented at reasonable cost. For high Reynolds number flows, we demonstrate that the numerical stabilization in the DG scheme acts as an implicit subgrid model. We examine the effect of the choice of the Reimann solver on the implicit subgrid scale effects. The initial results presented in this abstract focus on subsonic flows. The final version of the paper will include both subsonic and supersonic flows.

This abstract begins with a brief description of the governing equations and our space-time DG discretization in Section II. In Section III we present the Taylor-Green vortex problem which is used as a model problem throughout. In Section IV we discuss aliasing instabilities for marginally resolved simulations at moderate Reynolds number. In Section V we discuss nonlinear stability for under-resolved simulations at high- and infinite Reynolds number. In Section VI we discuss the implicit subgrid-scale modeling in underresolved high-Reynolds number flows and the effects of the choice of numerical flux. Finally, a summary and preliminary conclusions are presented in Section VII.

\section{Numerical Method}

The compressible Navier-Stokes equations are written in conservative form as:

$$
\begin{aligned}
\rho_{, t}+\left(\rho u_{i}\right)_{, i} & =0 \\
\left(\rho u_{j}\right)_{, t}+\left(\rho u_{i} u_{j}+p \delta_{i j}\right)_{, i} & =\tau_{i j, i} \\
(\rho E)_{, t}+\left(\rho u_{i} H\right)_{, i} & =\left(\tau_{i j} v_{j}+\kappa_{T} T_{, j} \delta_{i j}\right)_{, i}
\end{aligned}
$$

where $\rho$ is the density, $u_{j}$ are the components of the velocity, $E$ is the total energy, $p$ is the static pressure, $H=E+p / \rho$ is the total enthalpy, $\tau_{i j}$ is the viscous stress tensor, $\kappa_{T}$ is the thermal conductivity, $T=p / \rho R$ is the temperature, and $R$ is the gas constant. The pressure is given by:

$$
p=(\gamma-1)\left(\rho E-\frac{1}{2} \rho v_{k} v_{k}\right),
$$

where $\gamma$ is the specific heat ratio. The viscous stress tensor, $\tau_{i j}$, is given by:

$$
\tau_{i j}=\mu\left(v_{i, j}+v_{j, i}\right)-\lambda v_{k, k} \delta_{i j}
$$


where $\mu$ is the viscosity, $\lambda=\frac{2}{3} \mu$ is the bulk viscosity and $\delta_{i j}$ the Kronecker delta. We rewrite (1)-(3) as a single vector equation:

$$
\boldsymbol{u}_{, t}+\left(\boldsymbol{F}_{i}^{I}-\boldsymbol{F}_{i}^{V}\right)_{, i}=0
$$

where $\boldsymbol{u}=\left[\rho, \rho u_{j}, \rho E\right]$ is the conservative state vector, while $\boldsymbol{F}_{i}^{I}$ and $\boldsymbol{F}_{i}^{V}$ are, respectively, the inviscid and viscous fluxes. Applying a change of variables $\boldsymbol{u}=\boldsymbol{u}(\mathbf{v})$, where $\mathbf{v}$ are the entropy variables, the Navier-Stokes equations may be rewritten as:

$$
A_{0} \mathbf{v}_{, t}+A_{i} \mathbf{v}_{, x_{i}}-\left(K_{i j} \mathbf{v}_{, x_{j}}\right)_{, x_{i}}=0
$$

with symmetric $A_{0}=\boldsymbol{u}_{, \mathbf{v}}, A^{i}=\boldsymbol{F}_{i, \boldsymbol{u}}^{I} A_{0}=\boldsymbol{F}_{i, \mathbf{v}}^{I}$ and $K_{i j}=\boldsymbol{F}_{i, \boldsymbol{u}_{, x_{j}}}^{V} A_{0}=\boldsymbol{F}_{i, \mathbf{v}, x_{j}}^{V}{ }^{14}$ The entropy variables are:

$$
\mathbf{v}=\left[\begin{array}{c}
-\frac{s}{\gamma-1}+\frac{\gamma+1}{\gamma-1}-\frac{\rho E}{p} \\
\frac{\rho u_{j}}{p} \\
-\frac{\rho}{p}
\end{array}\right]
$$

where $s=\log \left(p / \rho^{\gamma}\right)$ is the entropy.

We proceed to discretize (6) or (7) as follows. The spatial domain, $\Omega$, is partitioned into non-overlapping hexahedral elements, $\kappa$, while the time is partitioned into time intervals (time-slabs), $I^{n}=\left[t^{n}, t^{n+1}\right]$. Define $\mathcal{V}_{h}=\left\{\boldsymbol{w},\left.\boldsymbol{w}\right|_{\kappa} \in[\mathcal{P}(\kappa \times I)]^{5}\right\}$, the space-time finite-element space consisting of piece-wise polynomial functions in both space and time on each element. The weak form of the governing equations is given by:

$\sum_{\kappa}\left\{\int_{I} \int_{\kappa}-\left(\boldsymbol{w}_{, t} \boldsymbol{u}+\boldsymbol{w}_{, x_{i}}\left(\boldsymbol{F}_{i}^{I}-\boldsymbol{F}_{i}^{V}\right)\right)+\int_{I} \int_{\partial \kappa} \boldsymbol{w}\left(\widehat{\boldsymbol{F}_{i}^{I} \boldsymbol{n}_{i}}-\widehat{\boldsymbol{F}_{i}^{V} \boldsymbol{n}_{i}}\right)+\int_{\kappa} \boldsymbol{w}\left(t_{-}^{n+1}\right) \boldsymbol{u}\left(t_{-}^{n+1}\right)-\boldsymbol{w}\left(t_{+}^{n}\right) \boldsymbol{u}\left(t_{-}^{n}\right)\right\}=0$

where the second and third integrals arise due to the spatial and temporal discontinuity, respectively, of the basis functions. $\widehat{\boldsymbol{F}_{i}^{I} \boldsymbol{n}_{i}}$ and $\widehat{\boldsymbol{F}_{i}^{V} \boldsymbol{n}_{i}}$ denote single valued numerical flux functions approximating, respectively, the inviscid and viscous fluxes at the spatial boundaries of the elements. In this work, the inviscid flux (unless otherwise noted) is computed using the Roe flux, ${ }^{17,18}$ while the viscous flux is computed using the method of Bassi and Rebay. ${ }^{19}$ When discretizing using conservative variables, we seek a solution $\boldsymbol{u} \in \mathcal{V}_{h}$ and require (9) be satisfied for all $\boldsymbol{w} \in \mathcal{V}_{h}$. Similarly, when discretizing using the entropy variables, we seek a solution $\mathbf{v} \in \mathcal{V}_{h}$ which satisfies the weak form (9) for all $\boldsymbol{w} \in \mathcal{V}_{h}$. The space, $\mathcal{V}_{h}$ is spanned by the tensor product of 1D nodal Lagrange basis functions defined at the Gauss-Legendre points.

Under exact integration and conditions on the choice of numerical flux function, the space-time DG discretization using entropy variables satisfies a discrete Clausius-Duhem inequality ensuring discrete satisfaction of the second law of thermodynamics. ${ }^{15}$ In particular, this allows for the proof of a nonlinear entropy-stability for the numerical scheme. ${ }^{15}$ In practice, integrals appearing in (9) are approximated with numerical quadrature rules. We consider a collocated scheme, (where solution points are used as quadrature points) and polynomial dealiasing rules using more accurate quadrature. The choice of quadrature rules is discussed further in Section IV. We consider both entropy variable formulations consistent with the nonlinear stability proof in Section V.

With the choice of basis and an appropriate quadrature rule to perform numerical integration, (9) gives a system of nonlinear equations which must solved for each time-slab. In this work we use a preconditioned Jacobian-free Newton-Krylov solver we have previously presented in Ref. 13.

\section{Taylor-Green Vortex Problem}

The Taylor-Green vortex evolution is used as a model problem for turbulent flow as it involves only periodic boundary conditions, no forcing and a simple initial condition. The flow is solved on an isotropic domain, which spans $[0,2 \pi L]$ in each coordinate direction. The initial conditions are given by:

$$
\begin{aligned}
u & =V_{0} \sin (x / L) \cos (y / L) \cos (z / L) \\
v & =-V_{0} \cos (x / L) \sin (y / L) \cos (z / L) \\
w & =0 \\
p & \left.=\rho_{0} V_{0}^{2}\left[\frac{1}{\gamma M_{0}^{2}}+\frac{1}{16}(\cos (2 x)+\cos (2 y))(\cos (2 z)+2)\right)\right]
\end{aligned}
$$


where $u, v$ and $w$ are the components of the velocity in the $x$-, $y$ - and $z$-directions, $p$ is the pressure and $\rho$ is the density. The Taylor-Green vortex flow is simulated using the compressible Navier-Stokes equations at Mach number $M_{0}=0.1$. The flow is initialized to be isothermal $\left(\frac{p}{\rho}=\frac{p_{0}}{\rho_{0}}=R T_{0}\right)$. Simulations are performed at a variety of Reynolds numbers $R e=\frac{\rho_{0} V_{0} L}{\mu}$.

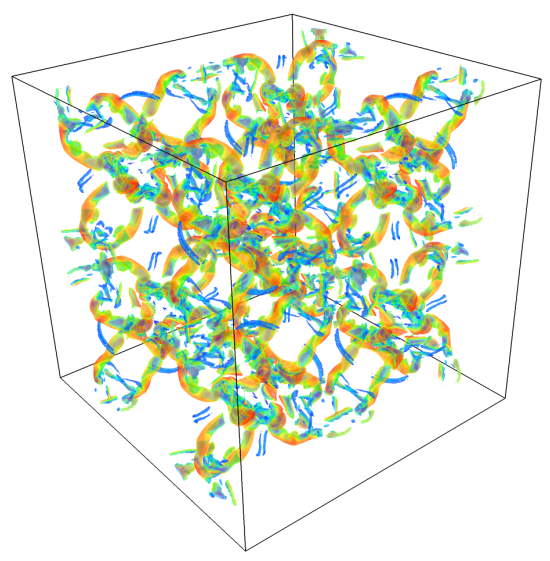

Figure 1. Iso-contours of vorticity magnitude at the instant of peak dissipation for the Taylor-Green vortex evolution at $M=0.1, R e=$ 1,600, computed using conservative variables, with $256^{3}$ degrees of freedom.

Starting from the simple initial condition, the flow becomes turbulent through repeated vortex stretching leading to progressively smaller eddies, which are then dissipated to heat through the action of molecular viscosity. With increasing Reynolds number, progressively smaller structures appear. Figure 1 shows the iso-contours of vorticity at the instant of peak dissipation from a 16th-order solution at $R e=1,600$.

For each simulation the temporal evolution of the kinetic energy

$$
E_{k}=\frac{1}{\Omega} \int_{\Omega} \frac{1}{2} \rho \mathbf{v} \cdot \mathbf{v} d \Omega
$$

is monitored. The evolution of the kinetic energy dissipation rate $\epsilon=-d E_{k} / d t$ was computed based on the data at the space-time quadrature points. We assess the quality of our numerical solutions by computing individual terms in the kinetic energy evolution equation. For compressible flow, the kinetic energy dissipation rate is given by the sum of three contributions $\epsilon=\epsilon_{1}+\epsilon_{2}+\epsilon_{3}=-d E_{k} / d t$ :

$$
\begin{aligned}
\epsilon_{1} & =\frac{1}{\Omega} \int_{\Omega} 2 \mu s_{i j} s_{i j} d \Omega \\
\epsilon_{2} & =\frac{1}{\Omega} \int_{\Omega} \lambda u_{k, k} u_{k, k} d \Omega \\
\epsilon_{3} & =-\frac{1}{\Omega} \int_{\Omega} p u_{k, k} d \Omega
\end{aligned}
$$

where $s_{i j}=\frac{1}{2}\left(u_{i, j}+u_{j, i}\right)$ is the strain rate tensor. Since the flow is nearly incompressible, we expect the dissipation due to the bulk viscosity, $\epsilon_{2}$, and the pressure-dilatation term, $\epsilon_{3}$, to be small. The kinetic energy dissipation rate is then approximately equal to $\epsilon \approx \epsilon_{1}$. However, for the compressible simulation this does not hold exactly. ${ }^{20}$

\section{Aliasing Errors}

We present initial validation of our numerical method by performing direct numerical simulation (DNS) of the Taylor-Green vortex problem at $R e=160$. The Reynolds number for this problem is sufficiently small that DNS may be performed with a relatively small number of degrees of freedom. We perform a 
mesh refinement study using our space-time DG scheme with 2nd-, 4th-, 8th-, 16th-order discretizations in space and 4th-order discretization in time. We use a formulation with conservative variables using both a collocated scheme and a polynomial dealiasing rule using $2 \mathrm{~N}$ points.

We perform a mesh refinement study using 48, 64, 96 and 128 degrees of freedom in each coordinate direction. The integral of the error in the dissipation rate as a function of the mesh size, $h=1 / D O F^{1 / 3}$, is presented in Figure 2. At low-order, (i.e. 2nd- and 4th-order) the numerical dissipation of the DG scheme plays a more significant role than the quadrature errors, so that the curves for collocated and dealiased schemes fall directly on top of each other. For these low Reynolds number simulations using the 16th-order scheme, the errors introduced through the use of collocated quadrature do not induce nonlinear instabilities. While not leading to nonlinear instabilities, the collocated scheme generates errors in the projection of the fluxes onto the polynomial space and large errors in the computed kinetic energy dissipation rate. In particular, using $48 \mathrm{DOF}$ in each coordinate direction, the inexact quadrature of the collocated 16th-order scheme results in an error in the computed kinetic energy dissipation rate which is an order of magnitude larger than the corresponding dealiased solution. With mesh refinement, both the solution and fluxes are well approximated in the polynomial space and thus the quadrature errors due to the collocated rule become less important. Both collocated and dealiased schemes demonstrate optimal error convergence rates, except at 16th-order where suboptimal convergence may be due to not yet having reached the asymptotic limit.

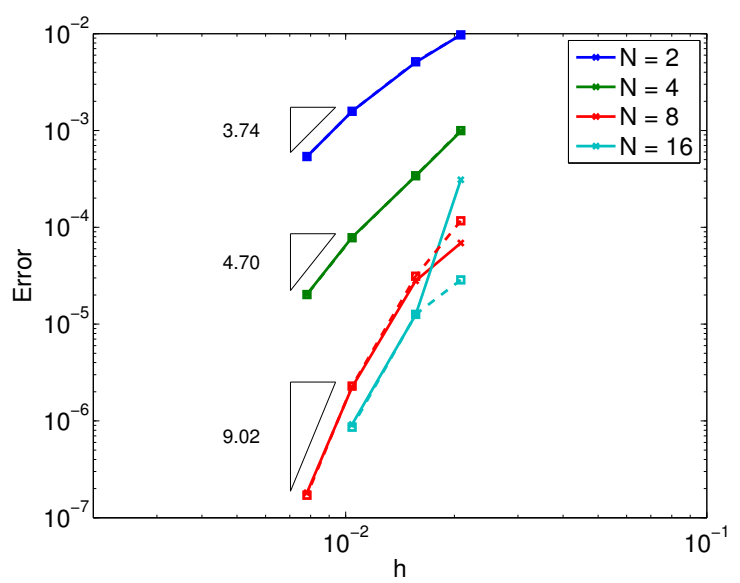

Figure 2. Error in kinetic energy evolution for Taylor-Green vortex problem at $M=0.1, R e=160$ computed using conservative variables. (solid line corresponds to the collocated scheme, dashed line corresponds to polynomial dealiasing using $2 \mathrm{~N}$ points

Next, we consider the Taylor-Green vortex problem at a larger Reynolds number of $R e=1,600$. Figure 3 shows the corresponding temporal evolution of the kinetic energy dissipation rate $-d E_{k} / d t$ computed with dealiased solutions using 256 degrees of freedom in each coordinate direction using 2nd- 4th-, 8th-, and 16th-order DG schemes. The corresponding meshes have, respectively, 128, 64, 32 and 16 elements in each coordinate direction. Reference data computed from an incompressible simulation using a spectral code on a $512^{3} \operatorname{grid}^{21}$ is also presented. For the 2nd-order scheme, there remains significant numerical dissipation at this resolution and the point of peak dissipation is poorly captured. With increasing solution order, the results relative to the spectral data are significantly improved. The dissipation rate for 4th-, 8th- and 16thorder schemes appear to fall directly upon those of the spectral data. However, zooming in at the point of peak dissipation, Figure 3(b) shows that 8th- and 16th-order schemes give somewhat better matching with the reference spectral data.

As we have seen with the lower Reynolds number case, higher-order schemes provide significant benefit for well resolved simulations. However, more often than not, turbulent flow simulations are marginally or underresolved. In these under-resolved simulations, higher-order schemes may provide benefit over lower-order schemes, however, due to the low numerical dissipation present in these schemes, nonlinear instabilities may arise if proper care is not taken. Gassner et al. ${ }^{12}$ demonstrated significant benefits in terms of computational efficiency using a explicit high-order DG scheme for computing a marginally resolved simulation of the 


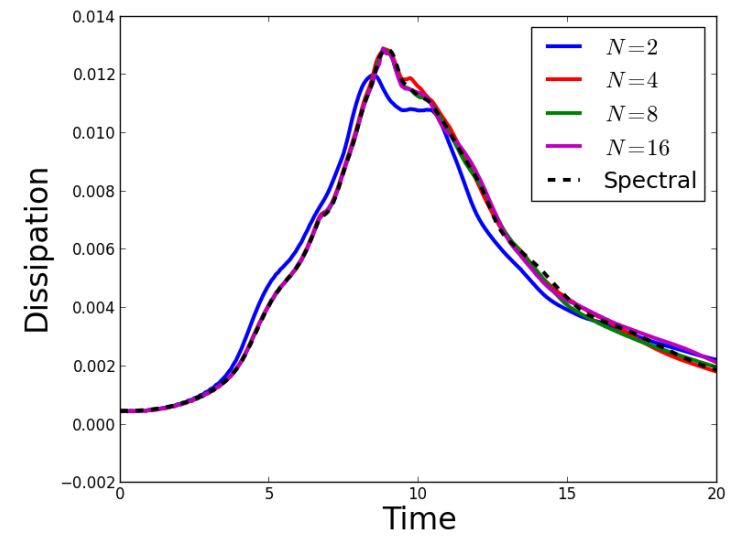

(a) Dissipation

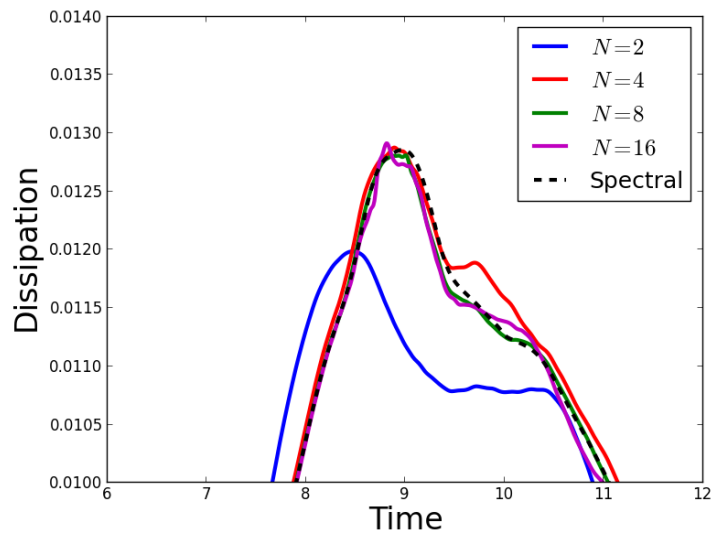

(b) Dissipation (Zoomed)

Figure 3. Taylor-Green vortex problem at $M=0.1, R e=\mathbf{1 , 6 0 0 , \text { computed }}$ with conservative variables using $256^{3}$ degrees of freedom.

Taylor-Green vortex at $R e=1,600$. In the following, we duplicate simulations of Gassner et al. ${ }^{12}$ using our space-time DG scheme. Figure 4(a) shows the evolution of the kinetic energy dissipation rate computed using a collocated DG scheme with a 4th-order temporal discretization and 2nd-, 4th-, 8th- and 16th-order spatial discretization using $64 \mathrm{DOF}$ in each coordinate direction. At 2nd- and 4th-order, the numerical dissipation present in the DG scheme results in significant kinetic energy dissipation and we show very poor agreement with the reference spectral data. However, the numerical dissipation is sufficient to maintain stability of the DG scheme. At 8th- and 16th-order, the aliasing errors present in the collocated scheme cause nonlinear instabilities which eventually lead to catastrophic failure of the simulations.

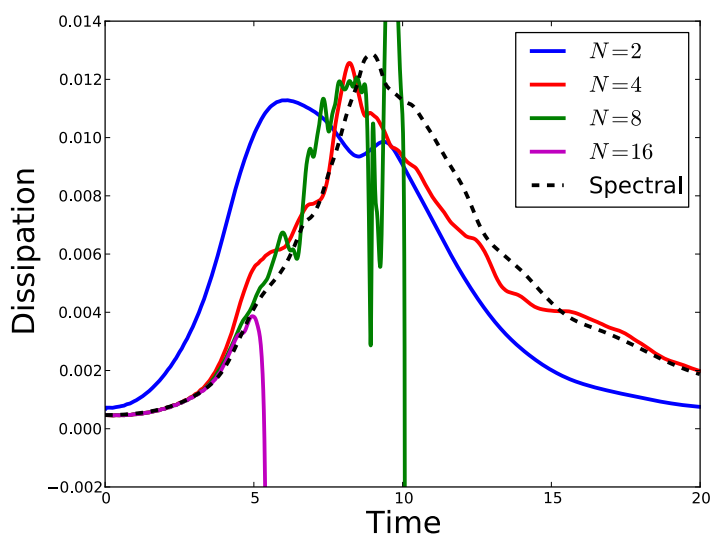

(a) Collocated

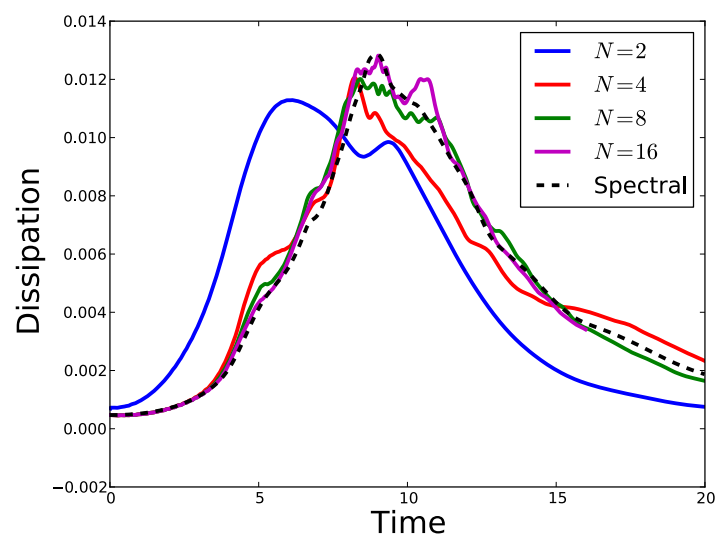

(b) Polynomial $2 N$ de-aliasing

Figure 4. Taylor-Green vortex problem at $M=0.1, R e=1,600$ computed using meshes with $64^{3}$ degrees of freedom using a) collocated quadrature, and b) polynomial de-aliasing with $2 N$ points

The corresponding evolution of the kinetic energy dissipation rate using a polynomial dealiasing with with $2 \mathrm{~N}$ quadrature points is shown in Figure 4(b). The use of a more accurate quadrature rule reduces the aliasing errors such that 8th- and 16th- order simulations do not suffer from the nonlinear instabilities seen with the collocated scheme. Even for this marginally resolved simulation, there is clear benefits in using the higher-order scheme as the 8th- and 16th- order schemes compute a kinetic energy dissipation rate which matches well with the reference spectral DNS despite using an order of magnitude fewer degrees of freedom in each coordinate direction. 


\section{Entropy Stability}

In the previous section, we have shown that nonlinear instabilities may be suppressed using polynomial dealiasing for marginally resolved higher-order simulations of turbulent flows at moderate Reynolds numbers. Next, we consider simulation of turbulent flows at even higher Reynolds numbers. In Figure 5(a) we show the evolution of the kinetic energy dissipation rate for a simulation of the Taylor-Green vortex problem at a Reynolds number of $R e=16,000$ using our space-time DG scheme using a polynomial dealiasing rule with $2 \mathrm{~N}$ points, discretized with conservative variables. A 4th-order temporal and 8th- and 16th-order spatial discretizations are used with $48 \mathrm{DOF}$ in each coordinate direction. Using a polynomial dealiasing rule with $2 \mathrm{~N}$ points is insufficient to suppress nonlinear instabilities at high order, leading to catastrophic failure of the simulations. Furthermore, using a dealiasing rule with $3 \mathrm{~N}$ or $4 \mathrm{~N}$ points (not shown) does not improve the situation. Clearly, at high-order, the dissipation in the DG scheme is insufficient to ensure the suppression of the nonlinear instabilities for under-resolved high Reynolds number turbulent simulations using conservative variables.

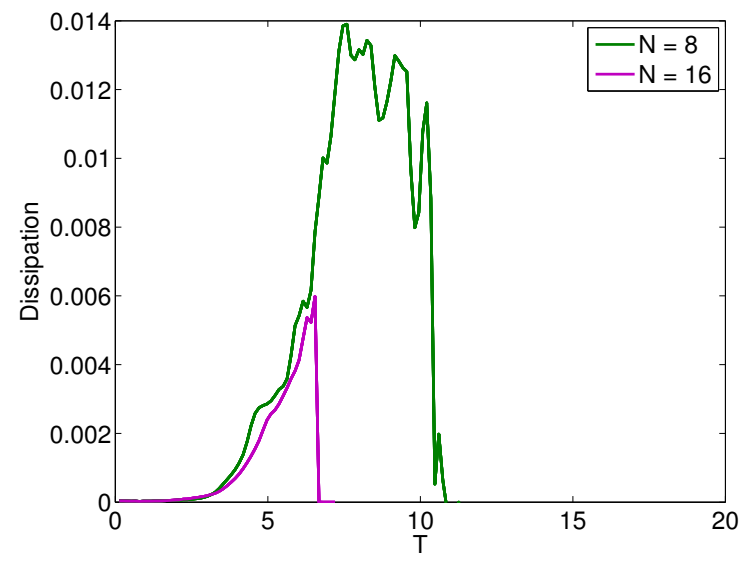

(a) Conservative Variables

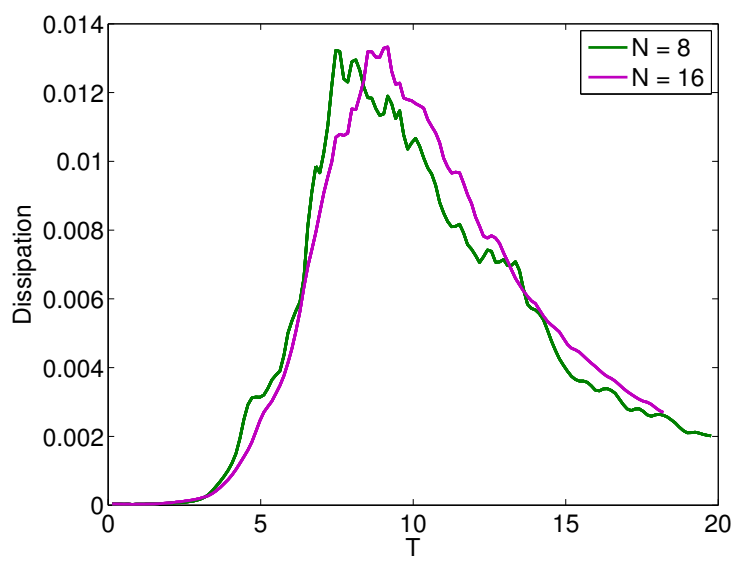

(b) Entropy Variables

Figure 5. Kinetic energy dissipation rate for the Taylor-Green vortex problem at $M=0.1, R e=16,000$ computed using meshes with $48^{3}$ degrees of freedom using polynomial dealiasing with $2 \mathrm{~N}$ points discretized with a) conservative variables and b) entropy variables

The corresponding kinetic energy dissipation rate, computed using the space-time DG scheme discretized with entropy variables and using a polynomial dealiasing rule with $2 \mathrm{~N}$ points, is shown in Figure 5(b). Using the entropy variables, the scheme is consistent with the nonlinear entropy stability proof and the 8thand 16th-order simulations do not exhibit nonlinear instabilities present when using conservative variables. The time history of entropy integrated over the domain using both conservative and entropy variables at 8th-order is presented in Figure 6. The simulations using conservative variables demonstrate non-physical entropy decay at the point of failure. On the other hand, simulations using entropy variables show monotonic entropy generation throughout the simulation.

\section{Implicit Sub-grid Modeling}

We have demonstrated the nonlinear stability properties of our space-time DG scheme discretized using entropy variables and polynomial dealiasing to perform under-resolved turbulent simulations at high Reynolds numbers. In this section we further demonstrate the nonlinear stability properties of our scheme by simulating the Taylor-Green vortex problem through infinite Reynolds number. We examine the sub-grid scale modeling effects of the DG scheme and examine the effect of the choice of numerical flux function.

First, we highlight the nonlinear stability of our space-time DG scheme by performing simulation of the Taylor-Green vortex problem at $R e=160,000$ using a 4th-order temporal and 16th-order spatial discretization on a sequence of meshes using $32,48,64,96$ and 128 DOF in each spatial direction. The kinetic energy dissipation rate for each simulation is plotted in Figure 7. With increasing mesh resolution, increasing frequency content is better represented on the discrete mesh and thus the peak of the computed kinetic energy 


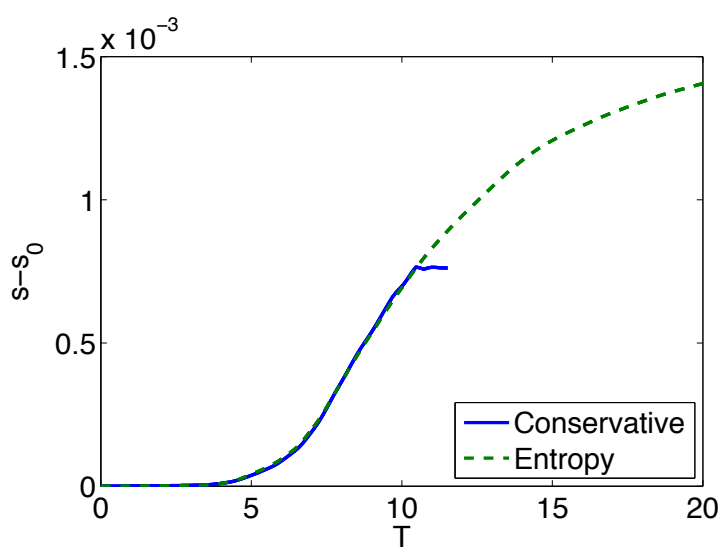

Figure 6. Entropy integrated over the domain for the Taylor-Green vortex problem at $M=0.1, R e=16,000$ computed using 8th-order spatial discretization with 48 degrees of freedom in each coordinate direction using polynomial dealiasing with $2 \mathrm{~N}$ points.

dissipation rate increases. The numerical dissipation present in the DG scheme acts as a subgrid scale model which reduces the effective Reynolds number.

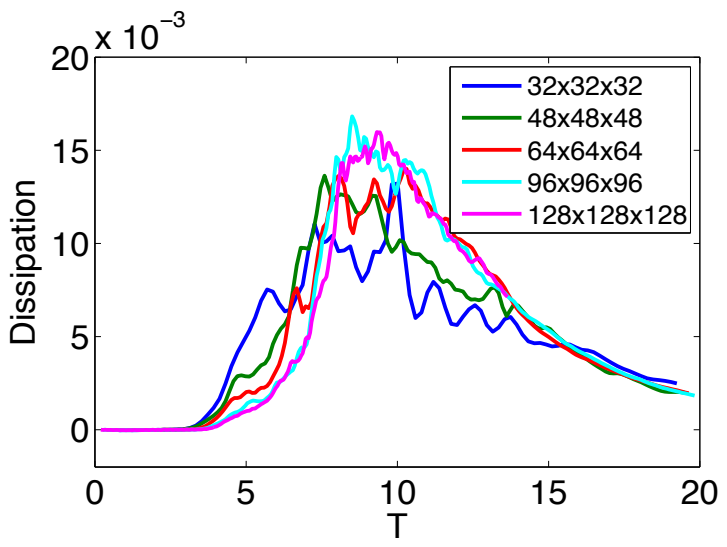

Figure 7. Kinetic energy dissipation rate for the Taylor-Green vortex problem at $M=0.1, R e=160,000$ computed using 8 th-order spatial discretization.

To investigate the subgrid scale effects of the numerical flux function, we perform simulations of the Taylor-Green vortex problem at moderate and infinite Reynolds number. (We note that higher-order simulations are only possible because of the nonlinear stability of the dealiased entropy variable formulation). We consider four numerical flux functions: the Roe flux, ${ }^{17}$ the flux of Ismail and Roe, ${ }^{18}$ the discrete symmetric mean-value flux, ${ }^{15}$ and the Lax-Friedrichs flux. The original flux function of Roe ${ }^{17}$ does not ensure discrete entropy production at an interface, ${ }^{15}$ and as such is not consistent with the nonlinear stability proof. The scheme of Ismail and Roe ${ }^{18}$ was developed to address this, and presented as a cheaper alternative to the discrete symmetric mean-value flux ${ }^{15}$ which requires numerical integration of the flux Jacobian between the two interface states. For our higher-order DG scheme, computation of numerical fluxes comprise a much smaller fraction of the total computational time than in a second-order finite volume formulations. Thus, the added cost of additional flux Jacobian evaluations required for the discrete symmetric mean-value flux is readily absorbed.

The evolution of the Taylor-Green vortex is simulated at $R e=1,600$ using the 8th-order DG scheme with the four different numerical flux functions. The kinetic energy dissipation rate, the dissipation due to viscous strain and the pressure-dilatation term are presented in Figure 8. The first three fluxes have identical linearized forms, thus we expect little difference between them for this subsonic flow which only involve small jumps across element interfaces. This is borne out by the simulations results. The Lax-Friedrichs flux shows 
significantly different behavior from the other three fluxes. In particular, using the Lax-Friedrichs flux results in large viscous dissipation due to strain. We note that all fluxes exhibit a positively biased dissipation due to the pressure-dilatation term as we have previously reported for our DG scheme. ${ }^{20}$

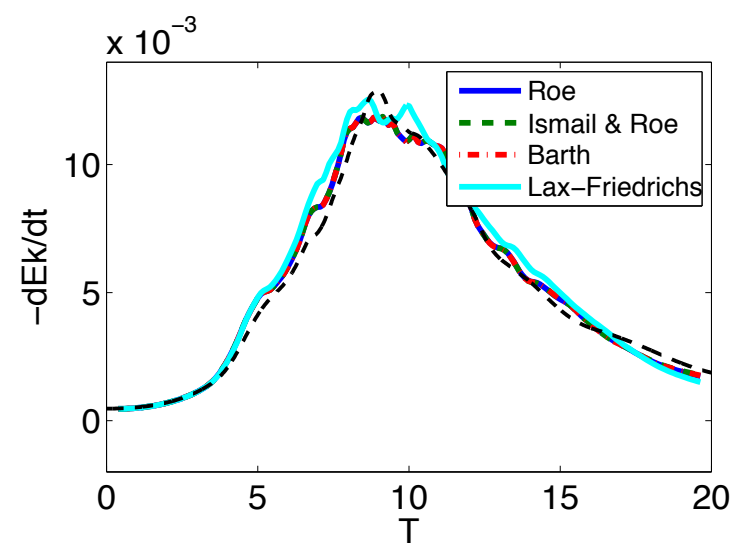

(a) Kinetic energy dissipation rate

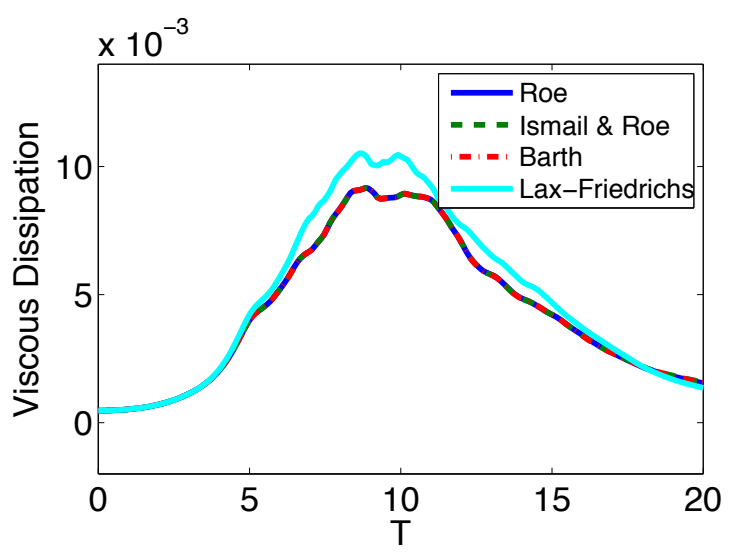

(b) Viscous Dissipation

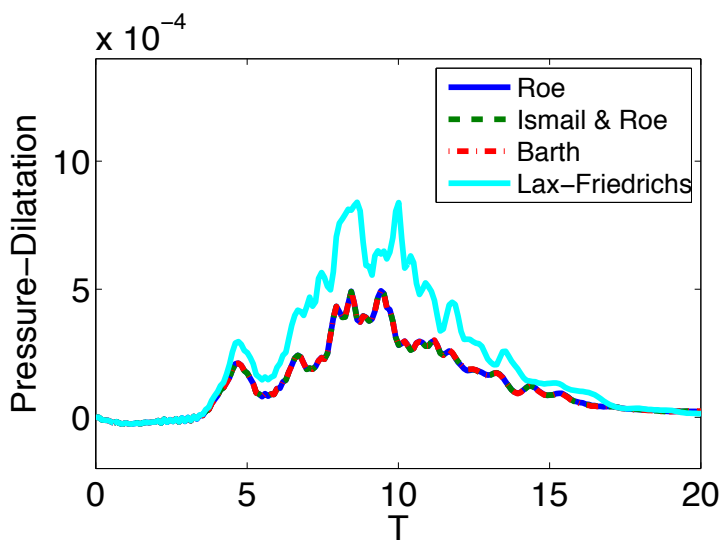

(c) Pressure Dilatation

Figure 8. Kinetic energy dissipation for the Taylor-Green vortex problem at $M=0.1, R e=1,600$ computed using 8th-order spatial discretization. (Flux options are Roe, ${ }^{17}$ Ismail and Roe, ${ }^{18}$ Barth, ${ }^{15}$ and Lax-Friedrichs

The kinetic energy spectrum using the flux of Ismail and Roe ${ }^{18}$ and the Lax-Friedrichs flux at a nondimensional time of $T=11.8$ in shown in Figure 9. The plots of the kinetic energy spectrum are consistent with the Lax-Friedrichs scheme having larger dissipation due to strain as there is more energy content at higher frequencies.

Next we perform simulations of the Taylor-Green vortex problem at infinite Reynolds number. We use our DG scheme with 4th-order temporal and 8th-order spatial discretizations. Figure 10 plots the kinetic energy spectrum using the flux of Ismail and $\operatorname{Roe}^{18}$ and the Lax-Friedrichs flux. For this infinite Reynolds number case, the Lax-Friedrichs flux exhibits a non-monotonic energy spectrum.

\section{Summary and Future Work}

We have presented a higher-order space-time discontinuous-Galerkin finite-element method. We have demonstrated the importance of formulating the DG scheme in a manner consistent with existing nonlinear entropy-stability theory. In particular, we have demonstrated that using entropy variables and a polynomial dealiasing rule is required to ensure nonlinear stability for under-resolved turbulent simulations at high-order. In this abstract we have demonstrated the subgrid-scale modeling effect of different numerical flux function formulations for subsonic flow. This abstract presented results on nonlinear stability for subsonic flows. The final version of the paper will include preliminary investigation into nonlinear stability for supersonic flows. 


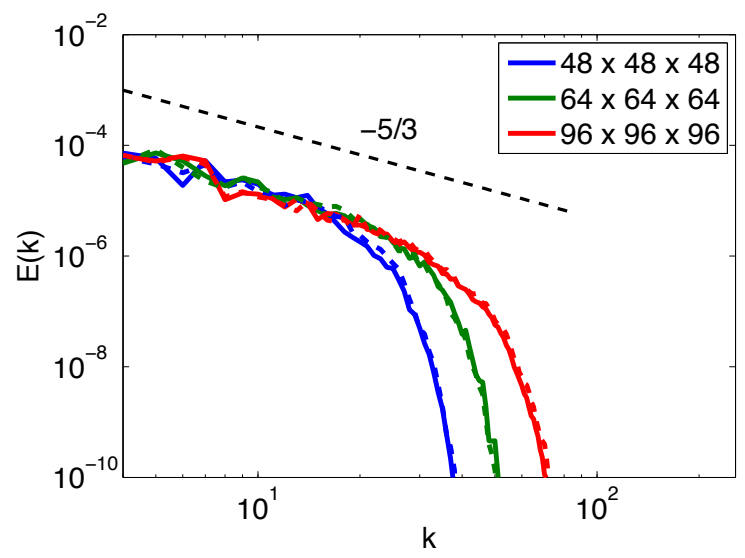

Figure 9. Kinetic energy spectrum for the Taylor-Green vortex problem at $M=0.1$, Re $=1,600$ computed using 8 thorder spatial discretization. (solid lines correspond to flux of Ismail and Roe, ${ }^{18}$ dashed line correspond to Lax-Friedrichs flux.)

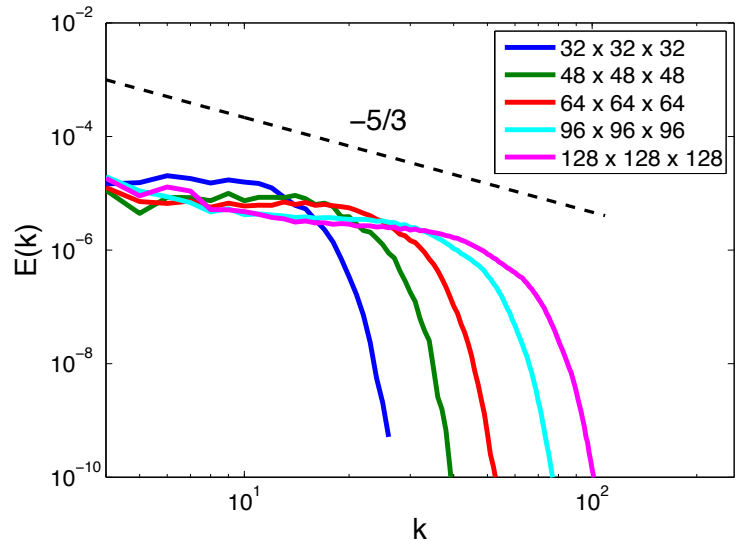

(a) Flux of Ismail \& Roe

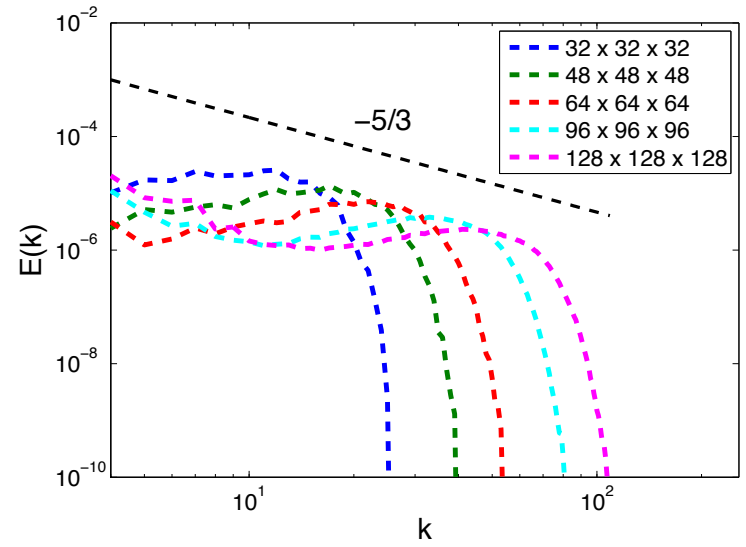

(b) Lax-Friedrichs flux

Figure 10. Kinetic energy spectrum for the Taylor-Green vortex problem at $M=0.1, R e=\infty$ computed using 8th-order spatial discretization. 
In particular, the final version of the paper will present analysis of the nonlinear stability of flux function formulations in combination with shock capturing schemes.

\section{References}

${ }^{1}$ Wang, Z., Fidkowski, K., Abgrall, R., Bassi, F., Caraeni, D., Cary, A., Deconinck, H., Hartmann, R., Hillewaert, K., Huynh, H., Kroll, N., May, G., Persson, P.-O., van Leer, B., and Visbal, M., "High-Order CFD Methods: Current Status and Perspective," International Journal for Numerical Methods in Fluids, Vol. 72, 2013, pp. 811-845.

${ }^{2}$ Orszag, S. and Patterson, G.S., J., "Numerical simulation of turbulence," Statistical Models and Turbulence, edited by M. Rosenblatt and C. Atta, Vol. 12 of Lecture Notes in Physics, Springer Berlin Heidelberg, 1972, pp. $127-147$.

${ }^{3}$ Morinishi, Y., Lund, T. S., Vasilyev, O. V., and Moin, P., "Fully Conservative Higher Order Finite Difference Schemes for Incompressible Flow," Journal of Computational Physics, Vol. 143, 1998, pp. 90-124.

${ }^{4}$ Honein, A. E. and Moin, P., "Higher entropy conservation and numerical stability of compressible turbulence simulations," Journal of Computational Physics, Vol. 201, 2004, pp. 531-545.

${ }^{5}$ Subbareddy, P. K. and Candler, G. V., "A fully discrete, kinetic energy consistent finite-volume scheme for compressible flows," Journal of Computational Physics, Vol. 228, No. 5, 2009, pp. 1347 - 1364.

${ }^{6}$ Sjgreen, B. and Yee, H., "On Skew-Symmetric Splitting and Entropy Conservation Schemes for the Euler Equations," Numerical Mathematics and Advanced Applications 2009, edited by G. Kreiss, P. Ltstedt, A. Mlqvist, and M. Neytcheva, Springer Berlin Heidelberg, 2010, pp. 817-827.

${ }^{7}$ Canuto, C., Hussaini, M., Quarteroni, A., and Zang, T., Spectral methods in fluid dynamics, Springer-Verlag, New York, NY, 1988.

${ }^{8}$ Pirozzoli, S., "Numerical Methods for High-Speed Flows," Annu. Rev. Fluid Mech., Vol. 43, 2011, pp. $163-194$.

${ }^{9}$ Tadmor, E., "Skew-selfadjoint form for systems of conservation laws," Journal of Mathematical Analysis and Applications, Vol. 103, No. 2, 1984, pp. $428-442$.

${ }^{10}$ Fisher, T. C. and Carpenter, M. H., "High-order Entropy Stable Finite Difference Schemes for Nonlinear Conservation Laws: Finite Domains," J. Comput. Phys., Vol. 252, Nov. 2013, pp. 518-557.

${ }^{11}$ Kirby, R. M. and Karniadakis, G. E., "De-aliasing on non-uniform grids: algorithms and applications," Journal of Computational Physics, Vol. 191, 2003, pp. 249-264.

${ }^{12}$ Gassner, G. and Beck, A. D., "On the accuracy of high-order discretizations for underresolved turbulence simulations," Theor. Comput. Fluid Dyn., Vol. 33, 2011, pp. 2560-2579.

${ }^{13}$ Diosady, L. T. and Murman, S. M., "Design of a variational multiscale method for turbulent compressible flows," AIAA Paper 2013-2870, 2013.

${ }^{14}$ Hughes, T. J. R., Franca, L., and Mallet, M., "A new finite element formulation for computational fluid dynamics: I Symmetric forms of the compressible Euler and Navier-Stokes equations and the second law of thermodynamics," Vol. 54, 1986, pp. 223-234.

${ }^{15}$ Barth, T. J., "Numerical Methods for Gasdynamic Systems on Unstructured Meshes," An Introduction to Recent Developments in Theory and Numerics for Conservation Laws, edited by D. Kroner, M. Olhberger, and C. Rohde, Springer-Verlag, 1999, pp. $195-282$.

${ }^{16}$ Beau, G. L., Ray, S., Aliabadi, S., and Tezduyar, T., "SUPG finite element computation of compressible flows with the entropy and conservation variables formulations," Computer Methods in Applied Mechanics and Engineering, Vol. 104, No. 3, 1993, pp. $397-422$.

${ }^{17}$ Roe, P. L., "Approximate Riemann solvers, parameter vectors, and difference schemes," Journal of Computational Physics, Vol. 43, No. 2, 1981, pp. 357-372.

${ }^{18}$ Ismail, F. and Roe, P. L., "Affordable, Entropy-consistent Euler flux functions II: entropy production at shocks," J. Comput. Phys., Vol. 228, No. 15, Aug. 2009, pp. 5410-5436.

${ }^{19}$ Bassi, F. and Rebay, S., "GMRES discontinuous Galerkin solution of the compressible Navier-Stokes equations," Discontinuous Galerkin Methods: Theory, Computation and Applications, edited by K. Cockburn and Shu, Springer, Berlin, 2000, pp. 197-208.

${ }^{20}$ Diosady, L. T. and Murman, S. M., "DNS of flows over periodic hills using a discontinuous Galerkin spectral element method," AIAA paper, 2014, accepted for publication.

${ }^{21}$ van Ress, W., Leonard, A., Pullin, D., and Koumoutsakos, P., "A comparison of vortex and pseudo-spectral methods for the simulation of periodic vortical flows at high Reynolds number," Journal of Computational Physics, Vol. 230, 2011, pp. 2794-2805. 